\title{
Temporal expression profiles of IncRNA and mRNA in human embryonic stem cell-derived motor neurons during differentiation
}

\author{
Xuejiao Sun ${ }^{1}$, Ming-Xing Li ${ }^{1}$, Chen-Zi Gong ${ }^{1}$, Jing Chen ${ }^{1}$, Mohammad Nasb ${ }^{1}$, Sayed Zulfiqar Ali Shah ${ }^{1}$, \\ Muhammad Rehan ${ }^{1}$, Ya-Jie Li ${ }^{1}$, Hong Chen ${ }^{\text {Corresp. } 1}$ \\ ${ }^{1}$ Department of Rehabilitation Medicine, Tongji Hospital, Tongji Medical College, Huazhong University of Science and Technology, Wuhan, China \\ Corresponding Author: Hong Chen \\ Email address: chenhong1129@hotmail.com
}

Background: Human embryonic stem cells (hESC) have are an invaluable research tool to study motor neuron development and disorders. However, transcriptional regulation of multiple temporal stages from ESCs to spinal motor neurons (MNs) has not yet been fully elucidated. Thus, the goals of this study were to profile the time-course expression patterns of IncRNAs during MN differentiation of ESCs and to clarify the potential mechanisms of the IncRNAs that are related to MN differentiation. Methods: We utilized our previous protocol which can harvest motor neuron in more than $90 \%$ purity from hESCs. Then, differentially expressed IncRNAs (DEIncRNAs) and mRNAs (DEmRNAs) during MN differentiation were identified through RNA sequencing. Bioinformatic analyses were performed to assess potential biological functions of genes. We also performed qRT-PCR to validate the DEIncRNAs and DEmRNAs. Results: Total 441 IncRNAs and 1068 mRNAs at day 6, 443 and 1175 at day 12, and 338 IncRNAs and 68 mRNAs at day 18 were differentially expressed compared with day 0 . Bioinformatic analyses identified that several key regulatory genes including POU5F1, TDGF1, SOX17, LEFTY2 and ZSCAN10, which involved in the regulation of embryonic development. We also predicted 283 target genes of DEIncRNAs, in which 6 mRNAs were differentially expressed. Significant fold changes in IncRNAs (NCAM1-AS) and mRNAs (HOXA3) were confirmed by qRT-PCR. Then, through predicted overlapped miRNA verification, we constructed IncRNA NCAM1-AS-miRNA-HOXA3 network. 


\section{Temporal expression profiles of IncRNA and mRNA in}

3 human embryonic stem cell-derived motor neurons during

4 differentiation

5

6

7

Xue-Jiao Sun ${ }^{1}$, Ming-Xing Li ${ }^{1}$, Chen-Zi Gong ${ }^{1}$, Jing Chen ${ }^{1}$, Mohammad Nasb ${ }^{1}$, Sayed Zulfiqar Ali Shah ${ }^{1}$, Rehan Muhammad ${ }^{1}$, Ya-Jie Li ${ }^{1}$, Hong Chen ${ }^{1, *}$

${ }^{1}$ Department of Rehabilitation Medicine, Tongji Hospital, Tongji Medical College, Huazhong University of Science and Technology, Wuhan, China

*Corresponding Author:

Hong Chen

1095 Jiefang Road, Wuhan 430030, China.

Email address: chenhong1129@hotmail.com

\section{Abstract}

Background: Human embryonic stem cells (hESC) are an invaluable research tool to study motor neuron development and disorders. However, transcriptional regulation of multiple temporal stages from ESCs to spinal motor neurons (MNs) has not yet been fully elucidated. Thus, the goals of this study were to profile the time-course expression patterns of lncRNAs during MN differentiation of ESCs and to clarify the potential mechanisms of the lncRNAs that are related to $\mathrm{MN}$ differentiation.

Methods: We utilized our previous protocol which can harvest motor neuron in more than $90 \%$ purity from hESCs. Then, differentially expressed lncRNAs (DElncRNAs) and mRNAs (DEmRNAs) during MN differentiation were identified through RNA sequencing. Bioinformatic analyses were performed to assess potential biological functions of genes. We also performed qRT-PCR to validate the DElncRNAs and DEmRNAs.

Results: Total 441 lncRNAs and 1068 mRNAs at day 6, 443 and 1175 at day 12, and 338 lncRNAs and 68 mRNAs at day 18 were differentially expressed compared with day 0 . Bioinformatic analyses identified that several key regulatory genes including POU5F1, TDGF1, SOX17, LEFTY2 and ZSCAN10, which involved in the regulation of embryonic development. We also predicted 283 target genes of DElncRNAs, in which 6 mRNAs were differentially expressed. Significant fold changes in lncRNAs (NCAM1-AS) and mRNAs (HOXA3) were confirmed by qRT-PCR. Then, through predicted overlapped miRNA verification, we constructed lncRNA NCAM1-AS-miRNA-HOXA3 network. 
39 Conclusion: This study comprehensively identified lncRNAs and mRNAs during MN

40 differentiation of hESCs, providing a theoretical basis for further study of the regulatory role of 41 lncRNAs in MN differentiation.

42

Keywords: Embryonic stem cells; Long non-coding RNA; Motor neuron; Differentiation; RNA sequencing

\section{Introduction}

Generation of specific cell types from hESCs in vitro have provided powerful platforms to study human disease and to understand fundamental biological processes. Highly efficient directed differentiation of hESCs into spinal motor neurons have been used to explore not only MN development but also MN disorders mechanism [1, 2]. Motor neurons are responsible for innervating skeletal muscles in the periphery and controlling movement. Transcription factors (TFs) regulate precise temporal and spatial gene expression in motor neuron specification and differentiation [3, 4]. The TFs Olig2 and Ngn2 function in opposition to regulate gene expression in $\mathrm{MN}$ progenitors in the pMN domain and the TFs Isl1 and Lhx3 are crucial for specifying MN identity [5-7].

LncRNAs, ranging in length from $200 \mathrm{nt}$ to $100 \mathrm{~kb}$, are highly expressed in the central nervous system. Accumulating evidence suggested that lncRNA played crucial roles in numerous biological and pathological processes at the chromatin remodeling level, transcriptional level and post-transcriptional level $[8,9]$. Notably, lncRNAs function as key regulators of cell differentiation and development, especially in neurogenesis. Particularly, lncRNAs can regulate ESC pluripotency and control multiple lineage differentiation by association with miRNAs, RNA-binding proteins, and epigenetic modifiers [10]. LncRNA-1604 functioned as competing endogenous RNAs (ceRNAs) of miR-200c and indirectly regulated the core TFs ZEB1 and ZEB2 during neural differentiation from mouse ESCs [11]. LncRNA Haunt functions as a genetic enhancer and an epigenetic repressor of HOXA gene activation during ESC differentiation [12]. Dlk1-Dio3 locus-derived lncRNAs play a critical role in maintaining postmitotic MN cell fate by repressing progenitor genes and they shape MN subtype identity by regulating Hox genes [13]. Nevertheless, at present very little functional characterization of lncRNAs in human motor neuron differentiation has been elucidated.

We used spinal motor neuron differentiation to profile the temporal changes without further purification steps. We combined highly efficient $\mathrm{MN}$ differentiation of hESCs in vitro with RNA-seq analysis to reveal the expression profiles of lncRNAs and mRNAs. Our findings may provide a new theoretical basis for further studies on lncRNAs modulation of motor neuron differentiation. 
75 Materials and Methods

76 Cell cultures form embryonic stems cells to spinal motor neurons

77 H9 (WA09, NIH registry 0046) hESC lines were obtained from WiCell Research Institute

78 (Madison, WI). Human ESCs were maintained on irradiated mouse embryonic fibroblasts and

79 differentiated as described before [2]. Briefly, ESCs were cultured using MN differentiation

80 medium containing DMEM/F12, Neurobasal medium, N2, B27, ascorbic acid, Glutamax and

81 penicillin/streptomycin (All from Gibco). The medium was additionally supplemented with

82 chemical compounds: $3 \mu \mathrm{M}$ CHIR99021 (Torcris), $2 \mu \mathrm{M}$ DMH-1 (Torcris) and $2 \mu \mathrm{M}$ SB431542

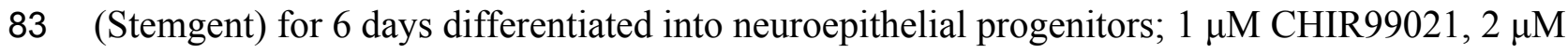

$84 \mathrm{DMH}-1$, and $2 \mu \mathrm{M}$ SB431542, $0.1 \mu \mathrm{M}$ RA (Sigma) and $0.5 \mu \mathrm{M}$ Purmorphamine (Pur, Sigma) for

856 days differentiated into MN progenitors; $0.5 \mu \mathrm{M}$ RA and $0.1 \mu \mathrm{M}$ Purmorphamine for 6 days

86 differentiated into MNs.

87

88

89

\section{Total RNA isolation and RNA sequencing}

Total RNA from samples was extracted using the Trizol reagent (Invitrogen). RNA quantity and

90

91

92 purity of total were confirmed using NanoDrop ND-2000 spectrophotometer (Thermo Fisher Scientific, USA). RNA integrity was assessed by Agilent 2100 Bioanalyzer (Agilent Technologies, USA). Ribosomal RNA was removed. Sequencing libraries were constructed. The dTTP were replaced by dUTP in the reaction buffer during second strand cDNA synthesis. Products were purified and library quality was assessed on the Agilent Bioanalyzer 2100 system. RNA Sequencing was performed on the Illumina HiSeq ((Illumina, USA) by KangChen database (accession number: GSE151744).

99

\section{Immunocytochemistry}

101

102

103

104

105

106

107

108

109

110

111

112

113

114

The primary antibodies information was used: SOX1 (gIgG, 1:1000, R\&D), HOXA3 (mIgG, 1:1000, R\&D), OLIG2 (rIgG, 1:1000, Millipore), HB9 (rIgG, 1:1000, Millipore), NF-200 (mIgG, 1:400, CST). Image J was used to perform cell counting (NIH, USA).

\section{Quantitative real-time PCR}

Total RNA was extracted using the Trizol reagent (Invitrogen). qRT-PCR was then performed and the $2^{-\Delta \Delta C t}$ method was calculated for quantification. The GAPDH was used as an internal control. The primer sequences used are listed in Table 1.

\section{Transfection of IncRNA Smart Silencer}

Motor neuron progenitors were transfected with lncRNA Smart Silencer (RiboBio Co., Guangzhou, China) to knock down the expression of NCM1-AS. The lncRNA Smart Silencer contained a mixture of three siRNAs and three antisense oligonucleotides. The target siRNA sequences were as followed: 5'-ACAACCCGATGACAGCAGA-3', 5'- 
115 CCAAATGGAGAACGTGCAA-3' and 5'-GTACTCGGTCTTTGCTGGC-3'. The target 116 antisense oligonucleotides sequence were as followed: 5'-ATGAAAGGAAAGGCACCAGC-3', 117 5'-ACATCTAACAAGGAGGACAC-3', 5'-AGGTTGACCGCAATGCACAT-3'. The negative

118 control (NC) Smart Silencer did not contain domains sequences homologous to those of humans, 119 rats, or mice. The cells were transfected with the lncRNA Smart silencer using Lipofectamine 1203000 (Invitrogen, USA) and collected 48 hours after transfection for RNA isolation.

121

122 Bioinformatic analysis

123 Gene Ontology (GO) analysis was used to investigate differentially expressed mRNAs with GO

124 categories. The predicted target genes above were conducted using the DAVID database

125 (http://david.abcc.ncifcrf.gov/). GO terms with a $P$ value $<0.05$ were considered as significantly 126 enriched. PPI networks was used STRING database (https://string-db.org/) and Cytoscape. The

127 networks were visualized in CytoHubb plug-in of Cytoscape. LncRNAs and mRNAs possessing 128 microRNA recognition elements (MREs) for the targeted miRNAs were predicted using the 129 miRanda and TargetScan.

130

131 Statistical analysis

132 All qRT-PCR results are expressed as the means \pm SEM of at least three independent

133 experiments. Statistical analyses were performed with SPSS statistics software version 22.0. P

134 values $<0.05$ was considered statistically significant. All graphs were made with GraphPad 135 Prism 8.

136 
137 Results

138 Differentiation of high purity motor neuron from human embryonic stem cells

139 In this study, we used hESCs to differentiate into spinal cord MNs in vitro. The MNs were

140 generated using chemical protocol described as method part [2] (Figure 1A). The hESCs can

141 differentiate into SOX1 neuroepithelial progenitors at day 6, OLIG2 motor neuron progenitors at

142 day 12, and HB9 motor neuron at day 18 high efficiently by using a combination of small

143 molecules (Figure 1B-D).

144

145

Patterns of gene expression changes from hESCs to motor neuron

146

High throughput sequencing is an efficient approach for investigating the biological function of

147

RNAs. All the differently expressed DElncRNAs and DElncRNAs were statistically significant

148 $(P<0.05)$ with fold change $(\mathrm{FC})>2$.

149

A total of 441 DElncRNAs (192 up-regulated and 249 down-regulated), 443 DElncRNAs (198

150

151 up-regulated and 245 down-regulated) and 338 DElncRNAs (164 up-regulated and 174 downregulated) were identified in D6 vs D0 (neuroepithelial progenitors, NEP), D12 vs D0 (motor

152 neuron progenitors, MNP), and D18 vs D0 (motor neuron, MN) respectively (Additional file 1). The volcano plots of expression profile for all the detected transcripts showed the relationship between the fold change and the significance (Figure 2A-C). To determine the key RNAs in human MN differentiation, we analyzed DElncRNAs in Venn diagram form. Veen analysis revealed that 33 lncRNAs were simultaneously up regulated and 78 were down regulated on D6, D12 and D18 (Figure 2D-E). Meanwhile, a total of 1068 DEmRNAs (360 up-regulated and 708 down-regulated), 1175 mRNAs (444 up-regulated and 731 down-regulated) and 68 DEmRNAs (34 up-regulated and 34 down-regulated) were identified in D6 vs D0, D12 vs D0 and D18 vs D0 respectively (Additional file 2). The volcano plots was shown in Figure 3A-C. Veen analysis revealed that 8 mRNAs were simultaneously up-regulated and 34 down-regulated (Figure 3D-E).

162

163

\section{Validation of IncRNAs and mRNAs expression}

To verify the results of the RNA sequencing, five strongly expressed DElncRNAs and

166

167

168

169

170

171

172

173

174

175

176

DEmRNAs during MN differentiation with FC $>40$ were selected for $\mathrm{qRT}$-PCR validation. As shown in Figure 4A-E, the expression of ZSCAN10, OCT4 and VRTN were down regulated, while HOXA3 and SP9 were up regulated. Moreover, the expression of ENST00000454596 (lncRNA rp11.001), ENST00000419695 (lncRNA rp11.003) and ENST00000583521 (lncRNA rp11-c9-001) were down regulated, while NCAM1-AS were up regulated (Figure 4F-J).

However, IncRNA H19 increased at D12, but decreased significantly at D18. Our results agreed with the data of RNA-sequencing generally.

\section{Bioinformatics analysis during MN differentiation}

To identify the key factors that regulated MN differentiation, GO analysis of DEmRNAs was performed on three different aspects namely biological process (BP), molecular function (MF) and cellular component (CC) shown in Figure 5A. In the BP domain, the top 6 GO terms were

Peer] reviewing PDF | (2019:09:41400:3:0:NEW 17 Aug 2020) 
177 enriched in cell migration involved in gastrulation, somatic stem cell population maintenance,

178

179

180

181

182

183

184

185

186

187

188

189

190

191

192

193

194

195

196

197

198

199

200

201

202

203

204

205

206

207

208

209

210

211

212

213

214

215

216

positive regulation of transcription from RNA polymerase II promoter, positive regulation of cell proliferation, cardiac cell fate determination and anterior/posterior pattern specification. Genes associated with cell migration involved in gastrulation were SOX17, MIXL1 and CER1. Genes associated with positive regulation of cell proliferation were EPHA1, ETS1, HOXA3, POU3F3, FLT1, and TDGF1. In the CC domain, the top 3 GO terms were transcription factor complex, membrane raft and nucleoplasm. In the MF domain, the top $3 \mathrm{GO}$ terms were sequence-specific DNA binding, transcription factor activity and HMG box domain binding. Figure 5B showed the heat map of DEmRNAs.

Furthermore, the Protein-Protein Interaction (PPI) network of DEmRNAs contained 28 nodes and 39 edges (Figure 5C). The topological analysis of the network was carried out by Network Analyzer in Cytoscape. PPI network was imported into Cytohubba to determine the hub transcription factors with high degree of connectivity between the nodes. The top ten hub genes were POU5F1, TDGF1, SOX17, LEFTY2, ZSCAN10, CER1, ZFP42, MIXL1, L1TD1 and ESRP1 shown in Figure 5C.

\section{Analysis of IncRNAs target mRNAs}

LncRNA may regulate nearby protein-coding genes by cis-regulatory effects. We analyzed potential function of the target genes of DElncRNAs at D18 vs D0. Total 338 DElncRNAs had 289 mRNAs target genes (Additional file 3). Then we performed GO analysis on DElncRNAs target genes to explore their potential biological functions. The GO terms related to BP, CC and MF were shown in Figure 6A. The most enriched BP term was related to positive regulation of telomerase activity. As for $\mathrm{CC}$, we found that the most enriched term were nucleoplasm and transcription factor complex. As for MF, transcription factor activity, sequence-specific DNA binding and metal ion binding were most enriched. Moreover, KEGG analysis was made in Figure 6B and the most enriched 5 top pathway terms were MAPK signaling pathway, Ribosome, Pyrimidine metabolism, Hippo signaling pathway and Cushing syndrome. Veen diagram analysis indicated that 6 mRNAs targeted by DElncRNAs were found at the interaction of DEmRNAs at D18 vs D0 (Figure 6C). Moreover, IncRNAs and their cis target DEmRNAs were up-regulated at D18, including two well-known TFs HOXA6 and HOXC9. We also performed the topological analysis of PPI network on these target genes (Figure 7), indicating the highly connected hub nodes in PPI network.

\section{Construction of IncRNA-miRNA-mRNA interaction network}

LncRNAs can regulate gene expression by acting as ceRNAs to sponge miRNAs [14].

Thererfore, we constructed a ceRNA interaction network from verified DE mRNAs and DE lncRNAs based on previous qRT-PCR data. As the RNA sequencing and PCR data shown, the transcription factors HOXA3 and SP9, exhibited continuous up-regulation in the transition from ESCs to MN stages, and especially showed a sharp up-regulation coincident with MNs specification. Interestingly, the expression of IncRNA NCAM1-AS showed same trend as 
217 HOXA3 and SP9. The NCAM1-AS is antisense lncRNA with two exons, produced from gene

218 NCAM1, which is involved in cell adhesion, axonal outgrowth, synapse formation during 219 development and differentiation, and highly expressed in the developing central and peripheral 220 nervous systems [15]. Bioinformatics analysis of same putative target miRNAs of HOXA3 and 221 lncRNA NCAM1-AS identified a direct binding site of has-miR-338-3p. Thus, lncRNA-miRNA222 mRNA pathway was constructed including: IncRNA NCAM1-AS-miR-338-3p-HOXA3. 223 Moreover, the expression of HOXA3 was down-regulated at D12 in the NCAM1-AS Smart 224 Silencer group compared to NC group (Figure 8A). Furthermore, immunofluorescence analysis 225 showed a decreased expression of OLIG2 in the NCAM1-AS knockdown group (Figure 8B). 226 Therefore, the results suggested that IncRNA NCAM1-AS can affect MNP differentiation.

227

228

\section{Discussion}

230

231

232

233

234

235

236

237

238

239

240

241

242

243

244

245

246

247

248

249

250

251

252

253

254

255

256

Motor neuron differentiation is precisely regulated and orchestrated by combinatorial expression of TFs during embryogenesis [4]. Accumulating evidence suggested that lncRNAs could interact with transcription factors to regulate cell differentiation [16-18]. In this study, we profiled mRNAs and lncRNAs expression from our highly efficient ESC-derived MN differentiation protocol to study the development of MNs. Our analysis focused on the identification of transcription factors and lncRNAs that are strongly involved in the temporal development of MNs.

ESCs, derived from the inner cell mass of blastocyst stage embryos, can both self-renew and differentiate into other cell types [19]. The balance between self-renewal and differentiation is regulated by a complex interaction network of translation factors. Pluripotent genes such as Oct4, Nanog, Sox2, Klf4, and Myc [20], activated in ESCs, were inhibited during cell differentiation, whereas expression of differentiation marker genes increases gradually.

Notably, we found the well-known pluripotency-associated transcription factors POU5F1 (also known as OCT4) and TDGF1 which were hub downstream-regulated genes upon MN differentiation in our study. It has been reported that lincRNA linc-RoR, may function as a key ceRNA to link the network of miRNAs and core TFs OCT4, SPX2, and NANOG, thus regulating ESC maintenance and differentiation [21]. As a previous study [22], hub transcription factor ZSCAN10, verified by qRT-PCR, also down-regulated in our study and could regulate ESCs gene expression and differentiation. OCT4 can directly regulate expression of ZSCAN10 and TDGF1 [23, 24].

Here, we also identified cis regulatory target genes HOXA6 and HOXC9 of lncRNA HOXAAS3 and HOXC-AS2 at MN stage, respectively. Spinal MNs acquire specialized pool identities that guide their axons to target muscles in the limb, and the specificity of these precise connections[25]. MNs could express many HOX genes specifying MN pool identity and connectivity [25, 26]. HOX6 paralog group genes (HOXA6, HOXC6, and HOXB6) contributed to diverse aspects of motor neuron subtype differentiation, and determined lateral motor column (LMC) fate at forelimb levels of the spinal cord [26]. In addition, HOXC9 determined thoracic 
257 level MN population fates, including preganglionic column (PGC) and hypaxial motor column 258 (HMC) neurons [27]. The LncRNA HOXA-AS3 was found to inhibit osteogenic differentiation 259 and promote adipogenic differentiation [28]. The up-regulated lncRNAs HOXA-AS3 and 260 HOXC-AS2 at human MN derived from ESCs was first identified in our study. The two 261 lncRNAs might be involved in MN differentiation by cis-regulating HOXA6 and HOXC9, which 262 needs further study.

263 Our sequencing results suggested a series of mRNAs and lncRNAs significantly changed 264 during the transition from ESC to motor neurons. HOXA3, SP9 and lncRNA NCAM1-AS 265 verified by PCR were observed dramatically up-regulated, especially at period of motor neuron. 266 HOXA3 and HOXB3 are necessary for the specification of Pax6-and Olig2-dependent somatic 267 MN progenitors [29]. In addition, HOX1 was reported to be involved in mediating both the role 268 of RA-signaling in specification of hindbrain MNs [30]. In our sequencing data, HOXB3 was up269 regulated at NEP and MNP stages but not altered at MN stage. The neuronal differentiation 270 marker NCAM was involved in motor neurons functionally expanding synaptic territory [31].

Additionally, the potential function of IncRNA NCAM1-AS has been originally identified in human MN differentiation from ESC. The lncRNAs could function as miRNA sponges and might compete against other endogenous RNAs to regulate mRNA expression levels and maintain normal biological function. Bioinformatics analysis indicated lncRNA-miRNA-mRNA pathway: IncRNA NCAM1-AS-miR-338-3p-HOXA3. A recent study found that miR-338-3p targeted and inhibited HOXA3 in breast cancer [32]. Thus, the upregulated IncRNA NCAM1-AS might inhibit the expression of miRNA by acting as a miRNA sponge, and in turn, increasing the expressions of MN differentiation-associated mRNAs HOXA3. The dysregulated lncRNAs may regulate gene expression through many ways and play a critical role in the processes of neuronal differentiation.

289 
290

291

292

293

294

295

296

297

298

299

300

301

302

303

304

305

306

307

308

309

310

311

312

313

314

315

\section{Declarations}

Acknowledgements

We acknowledge Professor Su-Chun Zhang of University of Wisconsin-Madison for his assistance during the development of this work.

\section{Competing interests}

The authors declare that they have no competing interests.

\section{Funding}

This study was supported in part by the National Key Research and Development Project of China (2016YFA0102500), the National Natural Science Foundation of China (81801066), the China Postdoctoral Science Foundation (2018M640706).

\section{Author contributions}

Xue-Jiao Sun was responsible for assembly of data, data analysis and interpretation, and manuscript writing. Ming-Xing Li and Chen-Zi Gong were responsible for the preliminary data search, selection and manuscript revision; Jing Chen and Ya-Jie Li, collection of data and data analysis. Mohammad Nasb, Sayed Zulfiqar Ali Shah and Rehan Muhammad were responsible for manuscript revision. Hong Chen were responsible for conception, experiment design and final approval of manuscript. All authors read and approval the final manuscript.

\footnotetext{
Author's information

Tongji Hospital, Tongji Medical College, Huazhong University of Science and Technology, Jiefang Avenue 1095, Wuhan 430030, China.
} 


\section{Reference}

318

2 Du ZW, H Chen, H Liu, J Lu, K Qian, CL Huang, X Zhong, F Fan, and SC Zhang. (2015). Generation and expansion of highly pure motor neuron progenitors from human pluripotent stem cells. Nat Commun 6: 6626.

3 Cave C and S Sockanathan. (2018). Transcription factor mechanisms guiding motor neuron differentiation and diversification. Curr Opin Neurobiol 53: 1-7.

4 Alaynick WA, TM Jessell, and SL Pfaff. (2011). SnapShot: spinal cord development. Cell 146: 178-178 e1.

5 Thaler JP, SK Lee, LW Jurata, GN Gill, and SL Pfaff. (2002). LIM factor Lhx3

contributes to the specification of motor neuron and interneuron identity through celltype-specific protein-protein interactions. Cell 110: 237-49.

6 Seo SY, B Lee, and S Lee. (2015). Critical Roles of the LIM Domains of Lhx3 in Recruiting Coactivators to the Motor Neuron-Specifying Is11-Lhx3 Complex. Mol Cell Biol 35: 3579-89.

7 Lee SK, B Lee, EC Ruiz, and SL Pfaff. (2005). Olig2 and Ngn2 function in opposition to modulate gene expression in motor neuron progenitor cells. Genes Dev 19: 282-94.

8 Mercer TR, ME Dinger, and JS Mattick. (2009). Long non-coding RNAs: insights into functions. Nature Reviews Genetics 10: 155-159.

9 Kopp F and JT Mendell. (2018). Functional Classification and Experimental Dissection of Long Noncoding RNAs. Cell 172: 393-407.

10 Loewer S, MN Cabili, M Guttman, YH Loh, K Thomas, IH Park, M Garber, M Curran, T Onder, S Agarwal, PD Manos, S Datta, ES Lander, TM Schlaeger, GQ Daley, and JL Rinn. (2010). Large intergenic non-coding RNA-RoR modulates reprogramming of human induced pluripotent stem cells. Nat Genet 42: 1113-7.

11 Weng R, C Lu, X Liu, G Li, Y Lan, J Qiao, M Bai, Z Wang, X Guo, D Ye, Z Jiapaer, Y Yang, C Xia, G Wang, and J Kang. (2018). Long Noncoding RNA-1604 Orchestrates Neural Differentiation through the miR-200c/ZEB Axis. Stem Cells 36: 325-336.

12 Yin Y, P Yan, J Lu, G Song, Y Zhu, Z Li, Y Zhao, B Shen, X Huang, H Zhu, SH Orkin, and X Shen. (2015). Opposing Roles for the lncRNA Haunt and Its Genomic Locus in Regulating HOXA Gene Activation during Embryonic Stem Cell Differentiation. Cell Stem Cell 16: 504-16.

13 Yen YP, WF Hsieh, YY Tsai, YL Lu, ES Liau, HC Hsu, YC Chen, TC Liu, M Chang, J Li, SP Lin, JH Hung, and JA Chen. (2018). Dlk1-Dio3 locus-derived lncRNAs perpetuate postmitotic motor neuron cell fate and subtype identity. Elife 7.

14 Thomson DW and ME Dinger. (2016). Endogenous microRNA sponges: evidence and controversy. Nat Rev Genet 17: 272-83.

15 Wobst H, B Schmitz, M Schachner, S Diestel, I Leshchyns'ka, and V Sytnyk. (2015). Kinesin-1 promotes post-Golgi trafficking of NCAM140 and NCAM180 to the cell surface. J Cell Sci 128: 2816-29.

361 Lopez-Pajares V, K Qu, J Zhang, DE Webster, BC Barajas, Z Siprashvili, BJ Zarnegar, LD Boxer, EJ Rios, S Tao, M Kretz, and PA Khavari. (2015). A LncRNA-MAF:MAFB 
362

363

364

365

366

367

368

369

370

371

372

373

374

375

376

377

378

379

380

381

382

383

384

385

386

387

388

389

390

391

392

393

394

395

396

397

398

399

400

401

402

403

404

405

406

407 transcription factor network regulates epidermal differentiation. Dev Cell 32: 693-706.

17 Ng SY, GK Bogu, BS Soh, and LW Stanton. (2013). The long noncoding RNA RMST interacts with SOX2 to regulate neurogenesis. Mol Cell 51: 349-59.

18 Wang P, Y Xue, Y Han, L Lin, C Wu, S Xu, Z Jiang, J Xu, Q Liu, and X Cao. (2014). The STAT3-binding long noncoding RNA lnc-DC controls human dendritic cell differentiation. Science 344: 310-3.

19 Thomson JA, J Itskovitz-Eldor, SS Shapiro, MA Waknitz, JJ Swiergiel, VS Marshall, and JM Jones. (1998). Embryonic stem cell lines derived from human blastocysts. Science 282: $1145-7$.

20 Chambers I and SR Tomlinson. (2009). The transcriptional foundation of pluripotency. Development 136: 2311-22.

21 Wang Y, Z Xu, J Jiang, C Xu, J Kang, L Xiao, M Wu, J Xiong, X Guo, and H Liu. (2013). Endogenous miRNA sponge lincRNA-RoR regulates Oct4, Nanog, and Sox2 in human embryonic stem cell self-renewal. Dev Cell 25: 69-80.

22 Zhang W, E Walker, OJ Tamplin, J Rossant, WL Stanford, and TR Hughes. (2006). Zfp206 regulates ES cell gene expression and differentiation. Nucleic Acids Res 34: 4780-90.

23 Wang ZX, CH Teh, JL Kueh, T Lufkin, P Robson, and LW Stanton. (2007). Oct4 and Sox2 directly regulate expression of another pluripotency transcription factor, Zfp206, in embryonic stem cells. J Biol Chem 282: 12822-30.

24 Babaie Y, R Herwig, B Greber, TC Brink, W Wruck, D Groth, H Lehrach, T Burdon, and J Adjaye. (2007). Analysis of Oct4-dependent transcriptional networks regulating selfrenewal and pluripotency in human embryonic stem cells. Stem Cells 25: 500-10.

25 Dasen JS, BC Tice, S Brenner-Morton, and TM Jessell. (2005). A Hox regulatory network establishes motor neuron pool identity and target-muscle connectivity. Cell 123: 477-91.

26 Lacombe J, O Hanley, H Jung, P Philippidou, G Surmeli, J Grinstein, and JS Dasen. (2013). Genetic and functional modularity of Hox activities in the specification of limbinnervating motor neurons. PLoS Genet 9: e1003184.

27 Jung H, J Lacombe, EO Mazzoni, KF Liem, Jr., J Grinstein, S Mahony, D Mukhopadhyay, DK Gifford, RA Young, KV Anderson, H Wichterle, and JS Dasen. (2010). Global control of motor neuron topography mediated by the repressive actions of a single hox gene. Neuron 67: 781-96.

28 Wu F, C Zhang, J Cai, F Yang, T Liang, X Yan, H Wang, W Wang, J Chen, and T Jiang. (2017). Upregulation of long noncoding RNA HOXA-AS3 promotes tumor progression and predicts poor prognosis in glioma. Oncotarget 8: 53110-53123.

29 Gaufo GO, KR Thomas, and MR Capecchi. (2003). Hox3 genes coordinate mechanisms of genetic suppression and activation in the generation of branchial and somatic motoneurons. Development 130: 5191-201.

30 Schubert M, ND Holland, V Laudet, and LZ Holland. (2006). A retinoic acid-Hox hierarchy controls both anterior/posterior patterning and neuronal specification in the developing central nervous system of the cephalochordate amphioxus. Dev Biol 296: 190-202.

31 Chipman PH, M Schachner, and VF Rafuse. (2014). Presynaptic NCAM is required for motor neurons to functionally expand their peripheral field of innervation in partially denervated muscles. J Neurosci 34: 10497-510. 
40832 Zhang L and F Ding. (2019). Hsa_circ_0008945 promoted breast cancer progression by 409 targeting miR-338-3p. Onco Targets Ther 12: 6577-6589.

410 


\section{Table $\mathbf{1}$ (on next page)}

Primer sequences of five IncRNAs and mRNAs 
Table 1 primer sequences of five IncRNAs and mRNAs

\begin{tabular}{lll}
\hline Gene & Forward & Reverse \\
GAPDH & GGAAGCTTGTCATCAATGGAAATC & TGATGACCCTTTTGGCTCCC \\
LncRNA rp11.001 & CAGCCCAAGGAACATCTCACC & TCTTGCCAACTTGAGTGTCCAT \\
LncRNA rp11.003 & ATCGGACTGTTCAACTCACCTG & TCAGCCGCTAAGCCAAGAAG \\
NCAM1-AS & TGAGATGCGAGACCTCCAGAC & CTCCAACTGCCTCATTATCCG \\
LncRNA rp11-c9-001 & GGGGGCTGGAAACCAACTTAT & CATCCCAAGTCCAGCGTGAA \\
H19 & CGGCCTTCCTGAACACCTTA & GTGTCTTTGATGTTGGGCTGATG \\
ZSCAN10 & GCCACCGTTTCCGCAATA & GCAGGTGTCGCAGCAGATT \\
CST1 & CCCCAAGGAGGAGGATAGGAT & AGTTGGGCTGGGACTTGGTA \\
VRTN & TCCCGCTCAACCTACTATGCC & CGTTTGAAGCAGCGATAGGG \\
OCT4 & TCTATTTGGGAAGGTATTCAGCC & CCTCTCACTCGGTTCTCGATACTG \\
HOXA3 & CTCAGAATGCCAGCAACAACC & ACAGGTAGCGGTTGAAGTGGA \\
SP9 & CCAAGCAGTTTTTCCGAGCAG & GGCTCGTGTTGCCGATCTT \\
\hline
\end{tabular}




\section{Figure 1}

Differentiation of hESCs into MNs

(A) Schematic diagram of the protocol used to obtain MNs from hESCs. hESCs, human embryonic stem cells; NEPs, neuroepithelial progenitors; MNPs, motor neuron progenitors; MNs, motor neurons. (B-D) hESCs differentiated to SOX1+ NEPs at day 6, OLIG2+ MNPs at day 12 and HB9+ MNs at day 18, respectively. Scale bar $=50 \mu \mathrm{m}$.

A

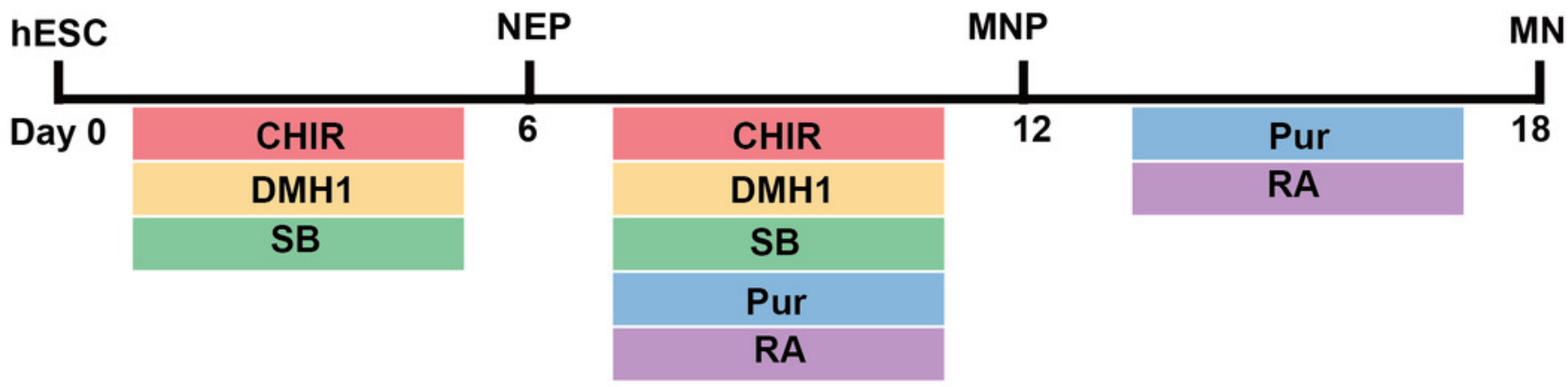

B

C

D

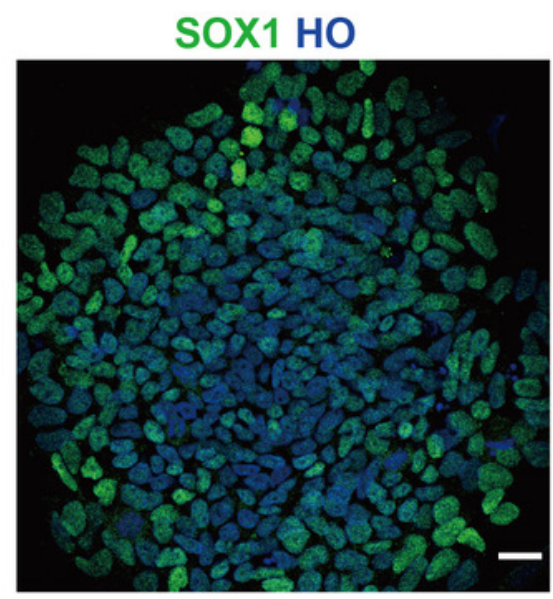

NEP

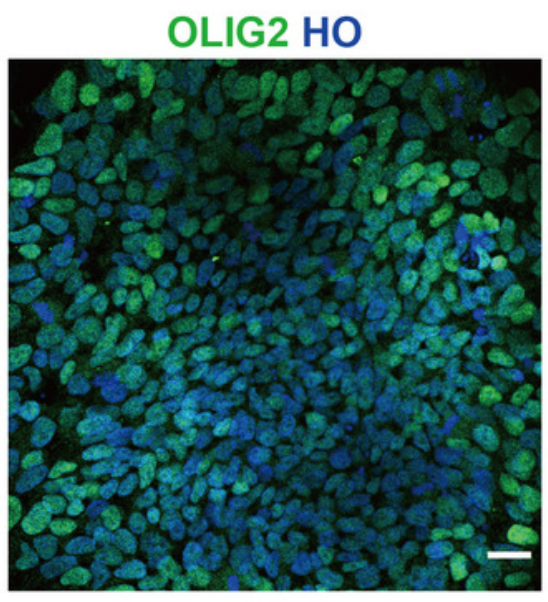

MNP

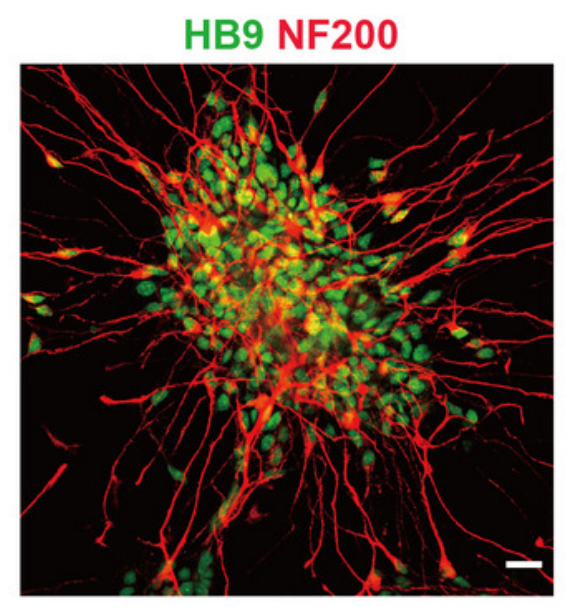

MN 
Figure 2

Expression Profiles of IncRNAs

(A-C) Plot indicated up-regulated and down-regulated IncRNAs at different stages points of MN differentiation from ESCs. (D-E) Venn diagram showed the number of overlap IncRNAs during the different stages of MN differentiation from ESCs.

A

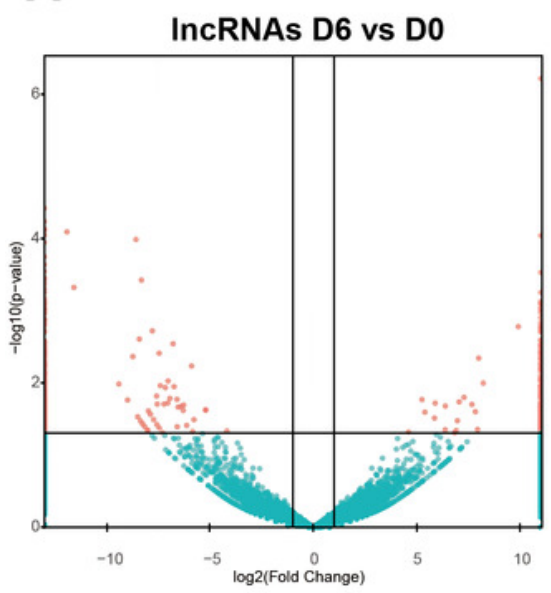

D

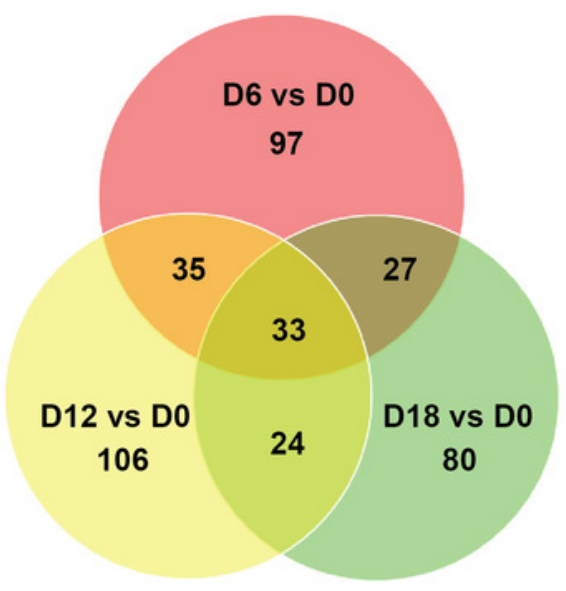

up-regulated IncRNAs
B

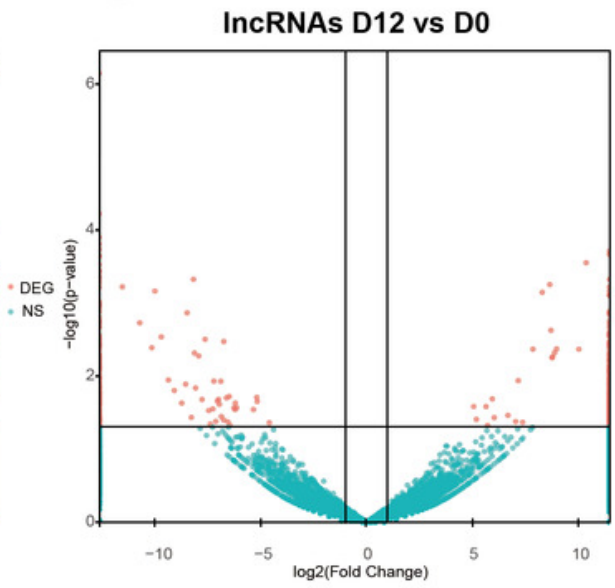

C

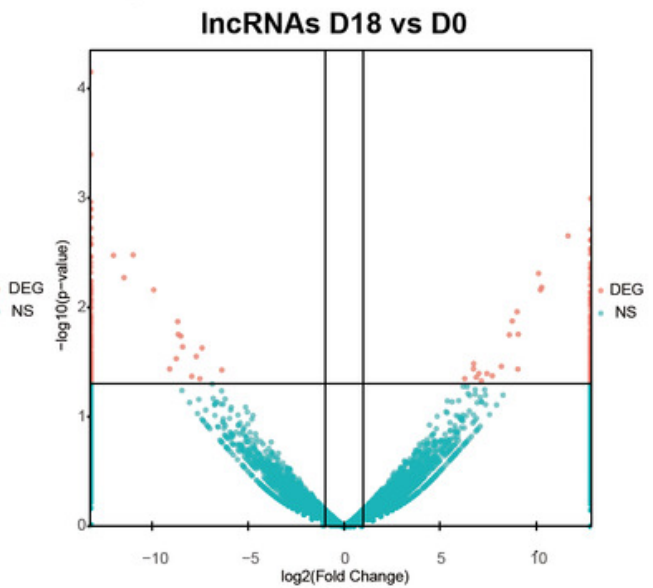

E

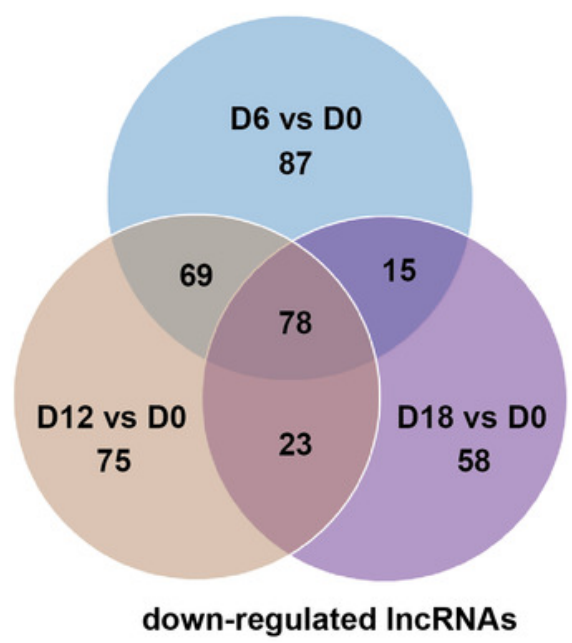


Figure 3

Expression Profiles of mRNAs

(A-C) Plot indicated up-regulated and down-regulated mRNAs at different stages points of MN differentiation from ESCs. (D-E) Venn diagram showed the number of overlap mRNAs during the different stages of MN differentiation from ESCs.
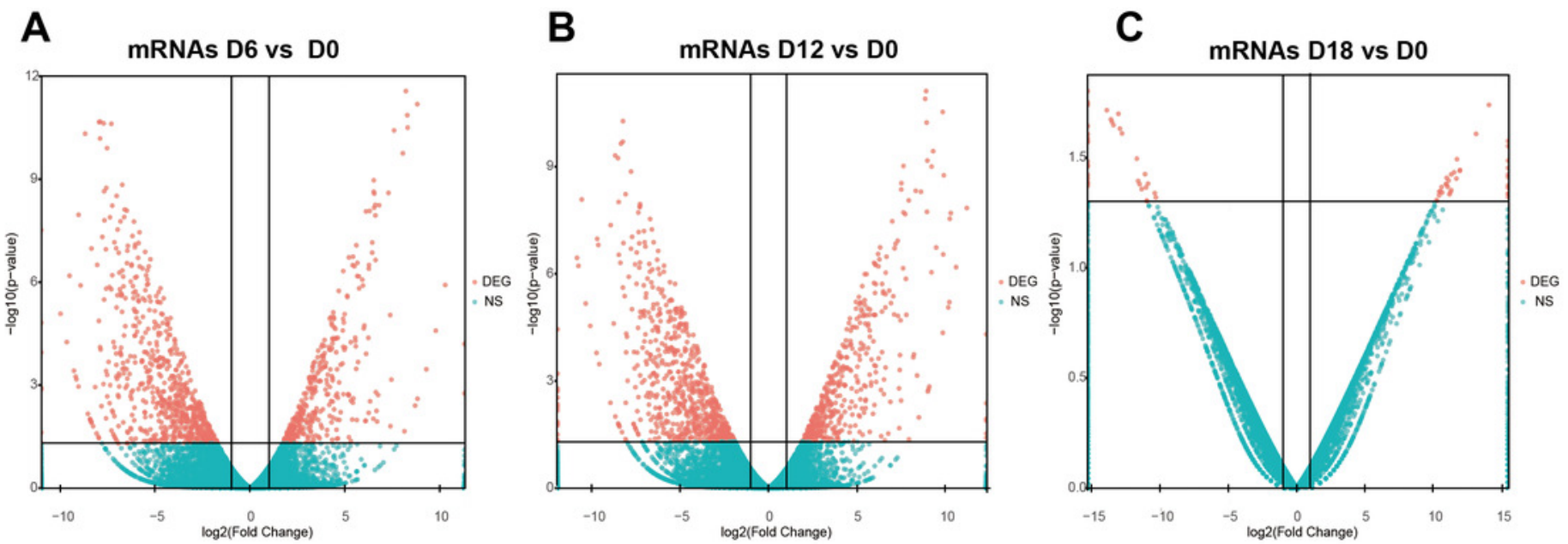

D

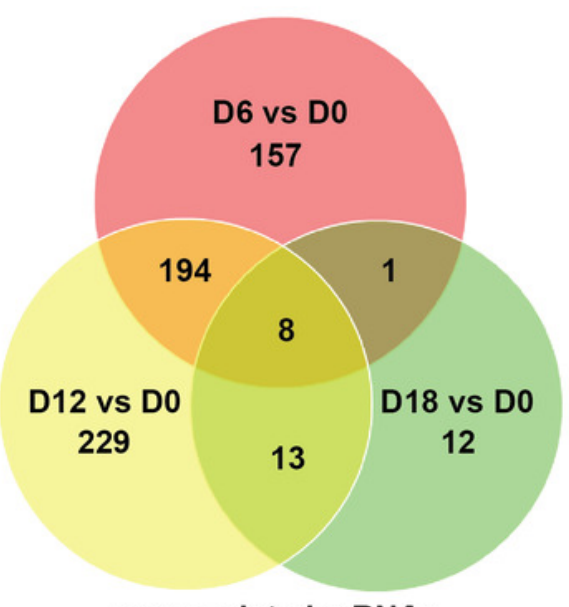

E

up-regulated mRNAs

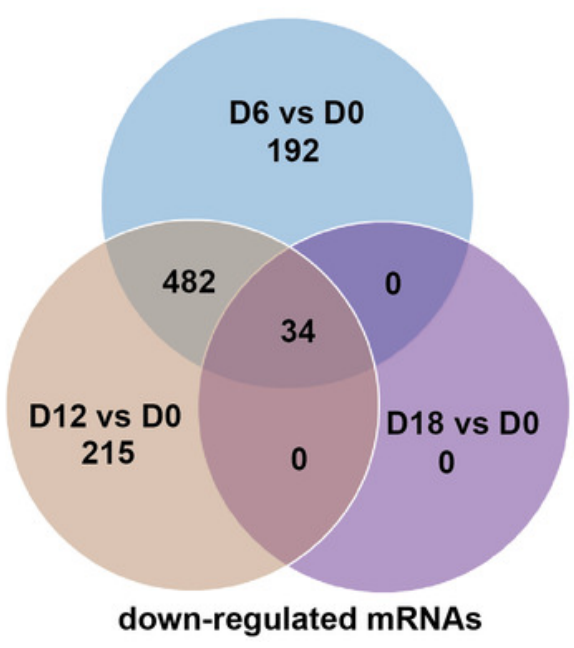


Figure 4

Validation of DEIncRNAs and mRNAs in MN differentiation

(A-E) qRT-PCR analysis of the 5 DEmRNAs during MN differentiation. (F-J) qRT-PCR analysis of the 5 DEIncRNAs up-regulated during $M N$ differentiation $* P<0.05$, ** $P<0.01$, *** $P<0.001$ and $* * * * P<0.0001$ compared to $\mathrm{D} 0$. The number of each group was three to four. 
A

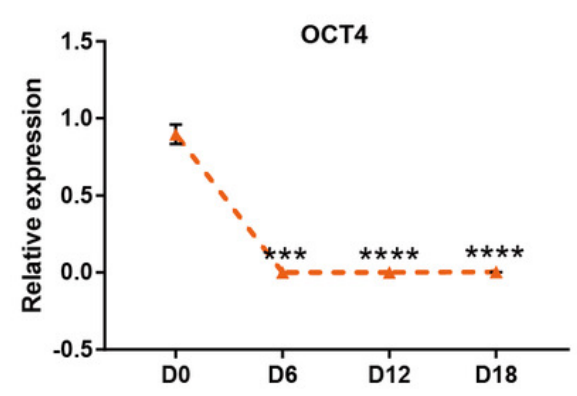

B

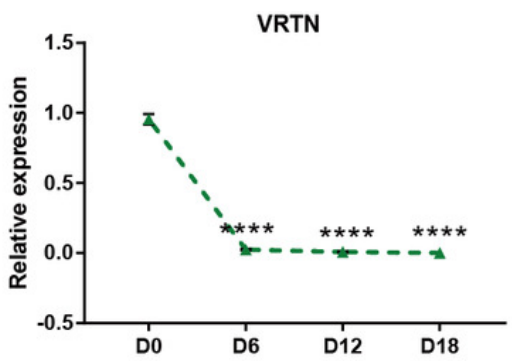

C

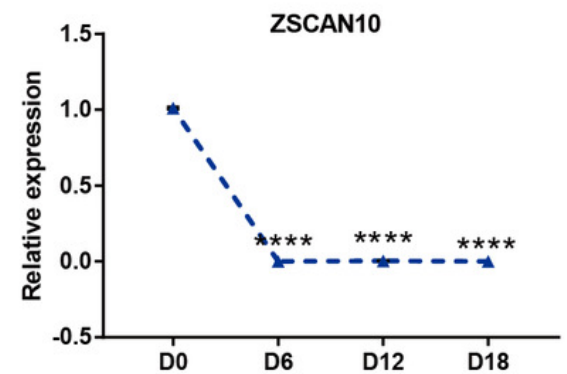

D

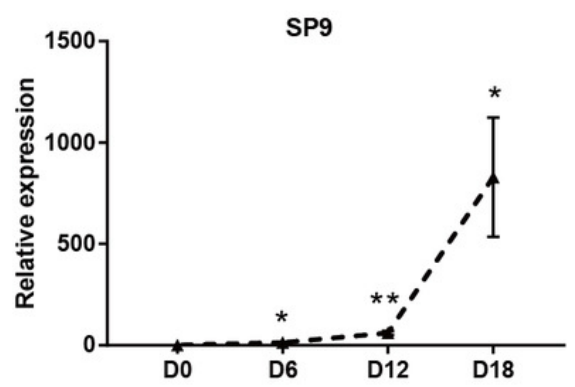

E

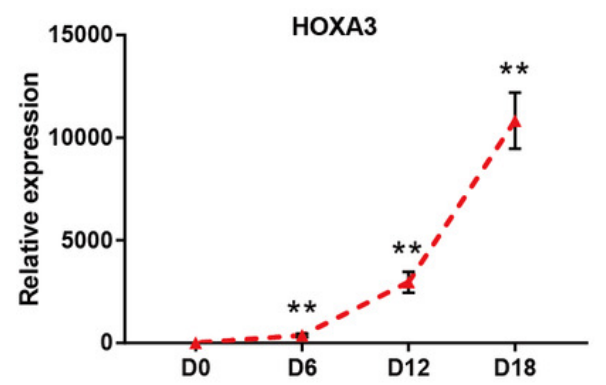

$\mathbf{F}$

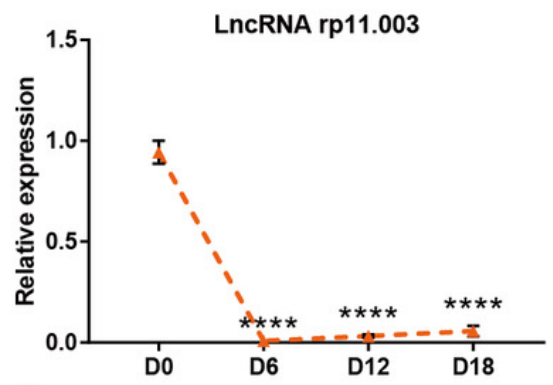

G

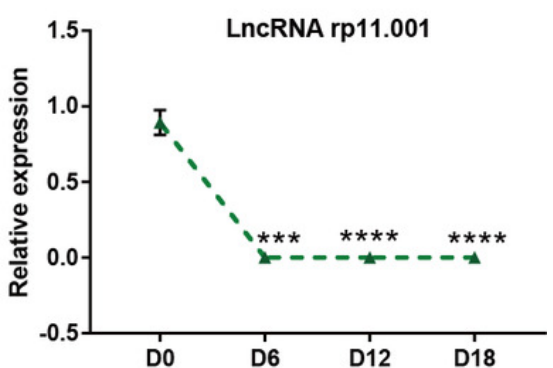

H
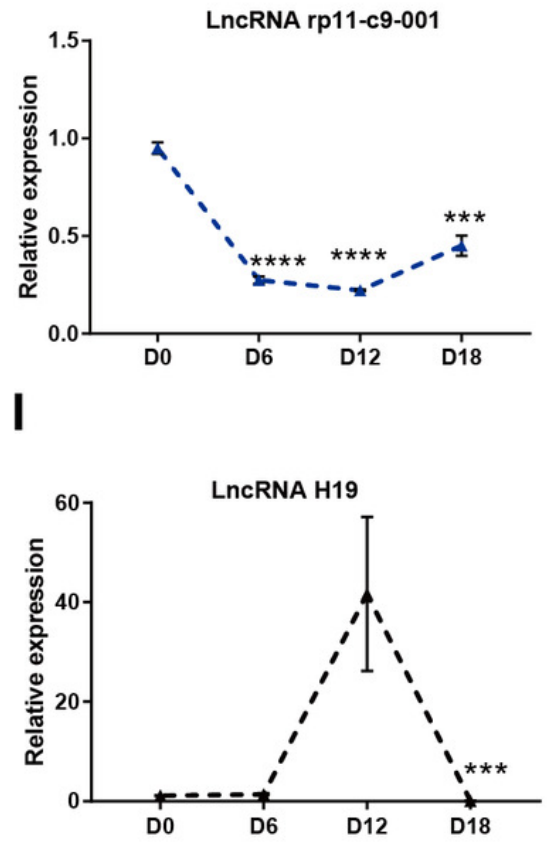

$\mathbf{J}$

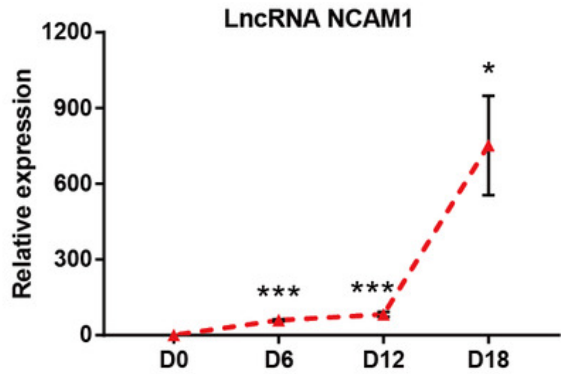

Peer) reviewing PDF | (2019:09:41400:3:0:NEW 17 Aug 2020) 


\section{Figure 5}

Bioinformatic analysis of DEmRNAs the biological function

(A) Go analysis of shared DEmRNAs at D6, D12 and D18 compared with D0. (B) Heat map of shared DEmRNAs at D6, D12 and D18 compared with D0. (C) Protein interaction network analysis of shared DE mRNAs at D6, D12 and D18 compared with D0. 
A

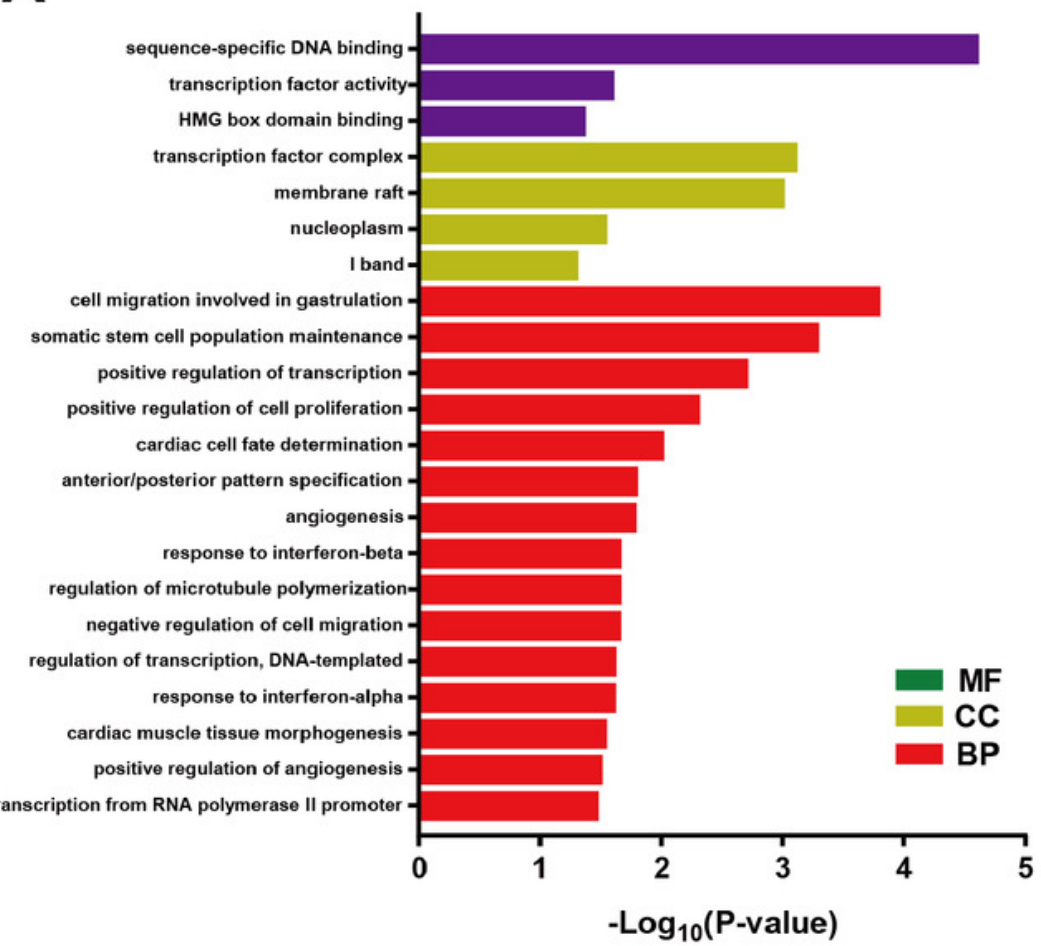

C

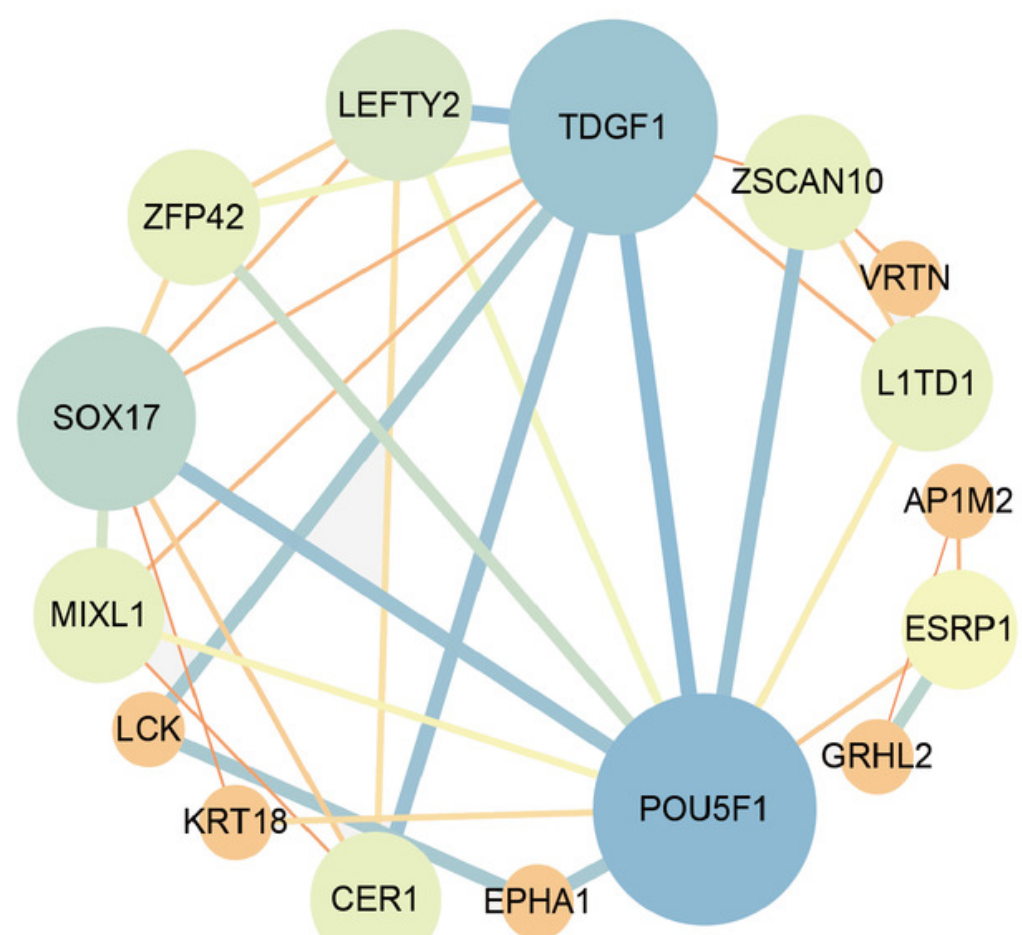

B

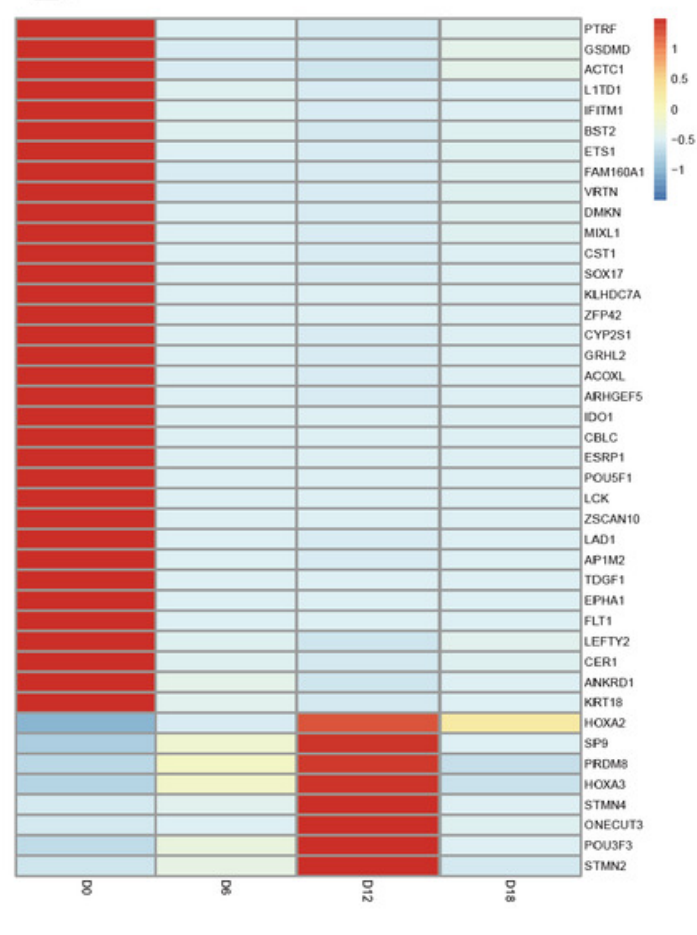

FLT1 ANKRD1 STMN4

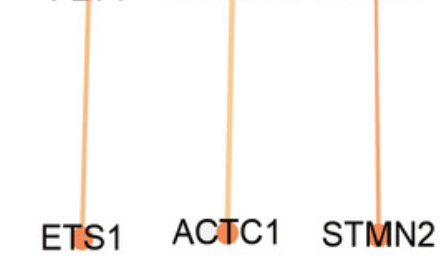

HOXA2 PRDM8 BST2

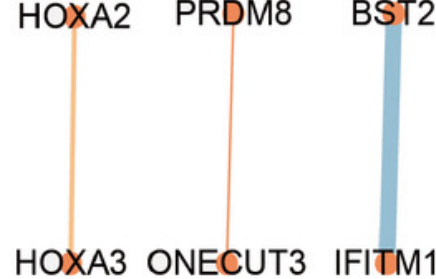


Figure 6

Bioinformatic analysis of DEIncRNAs the biological function

(A) Go analysis of DElncRNAs at MN stage. (B) KEGG analysis of DEIncRNAs at MN stage. (C) Veen diagram showed mRNAs tagerted by DEIncRNA and DEmRNAs at MN stage.

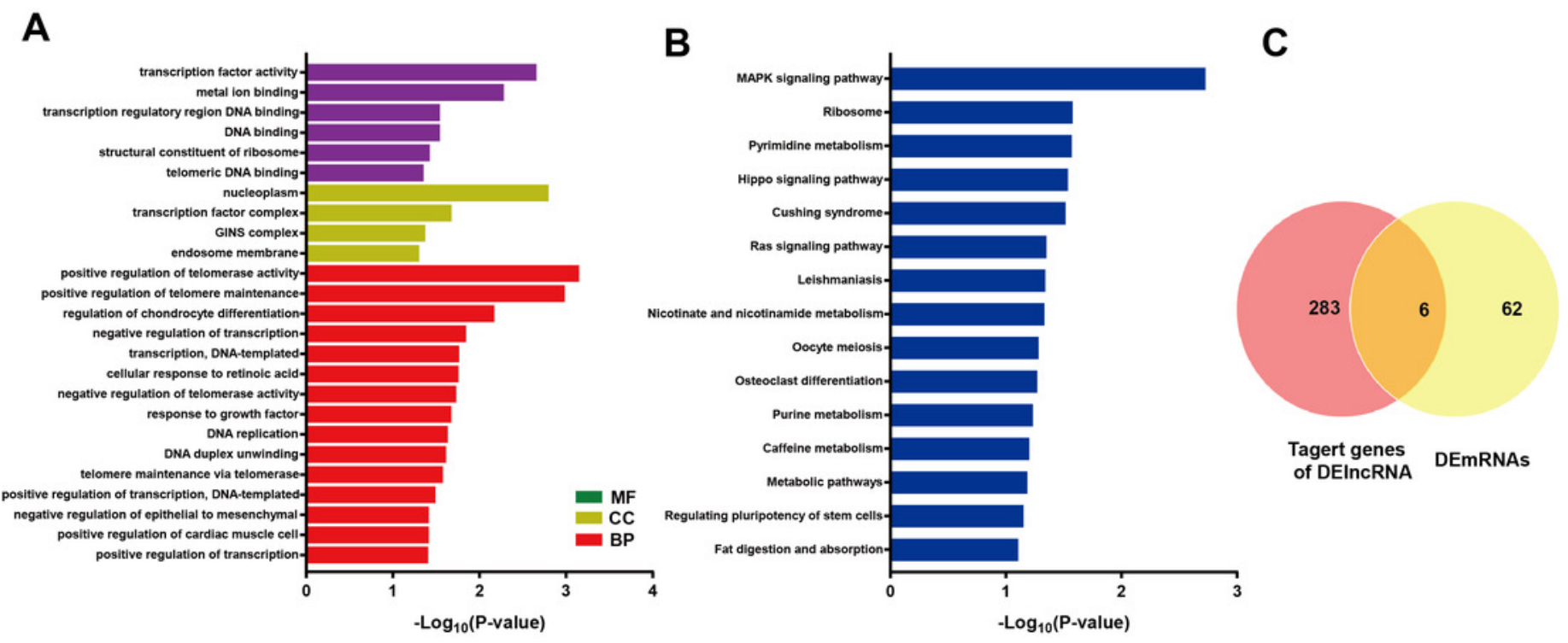


Figure 7

\section{Protein interaction network analysis of mRNAs targeted by DEIncRNAs at MN stage}

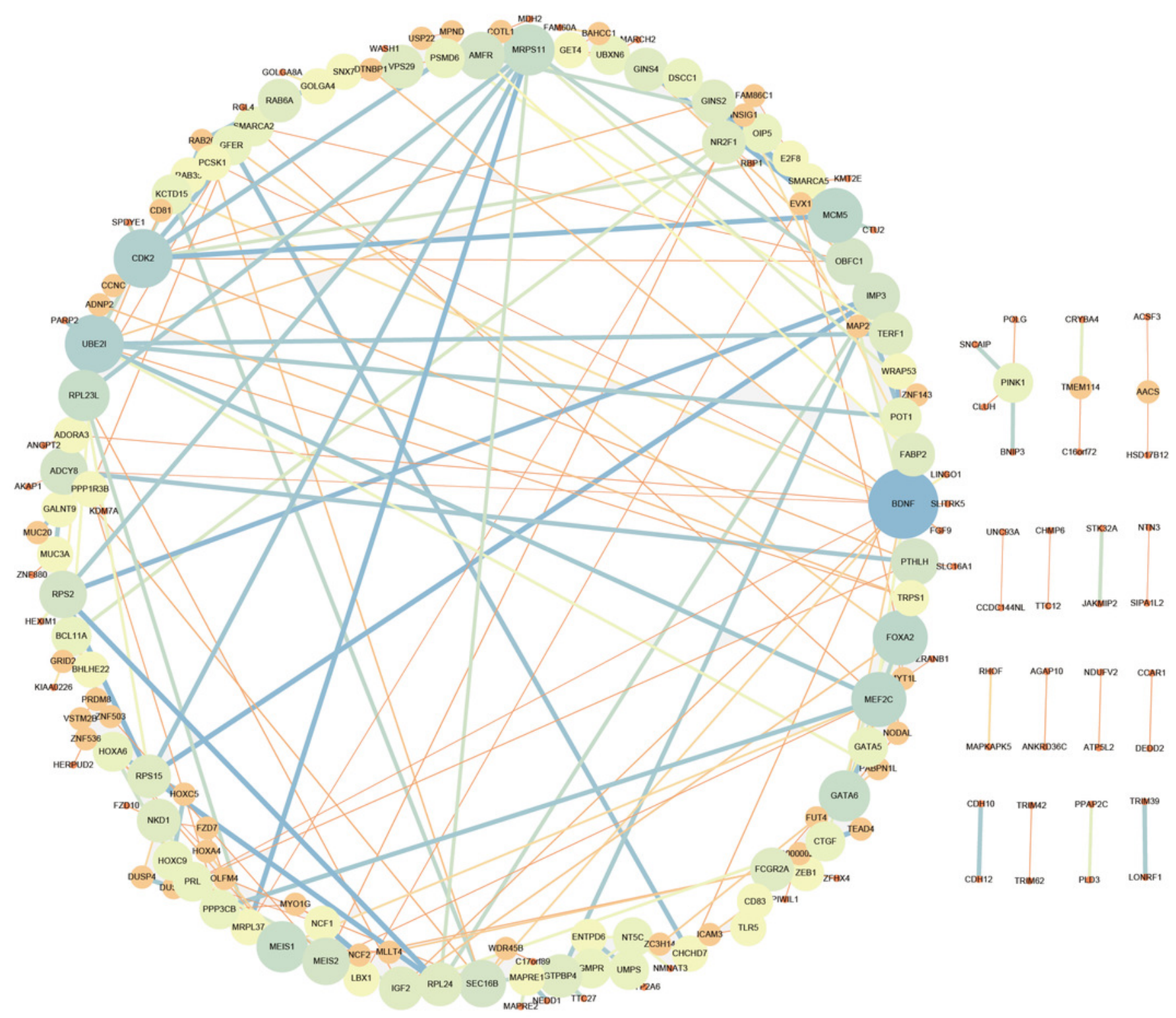




\section{Figure 8}

Figure 6. Silencing of IncRNA NCAM1-AS inhibit MNP differentiation.

(A) The expression of HOXA3 was analyzed after NCAM1-AS knockdown. (B) Immunostaining of OLIG2 expression in the after NCAM1-AS knockdown at MNP stage. Scare bar $=50 \mu \mathrm{m}$. $* \mathrm{P}$ $<0.05, * * P<0.01$.

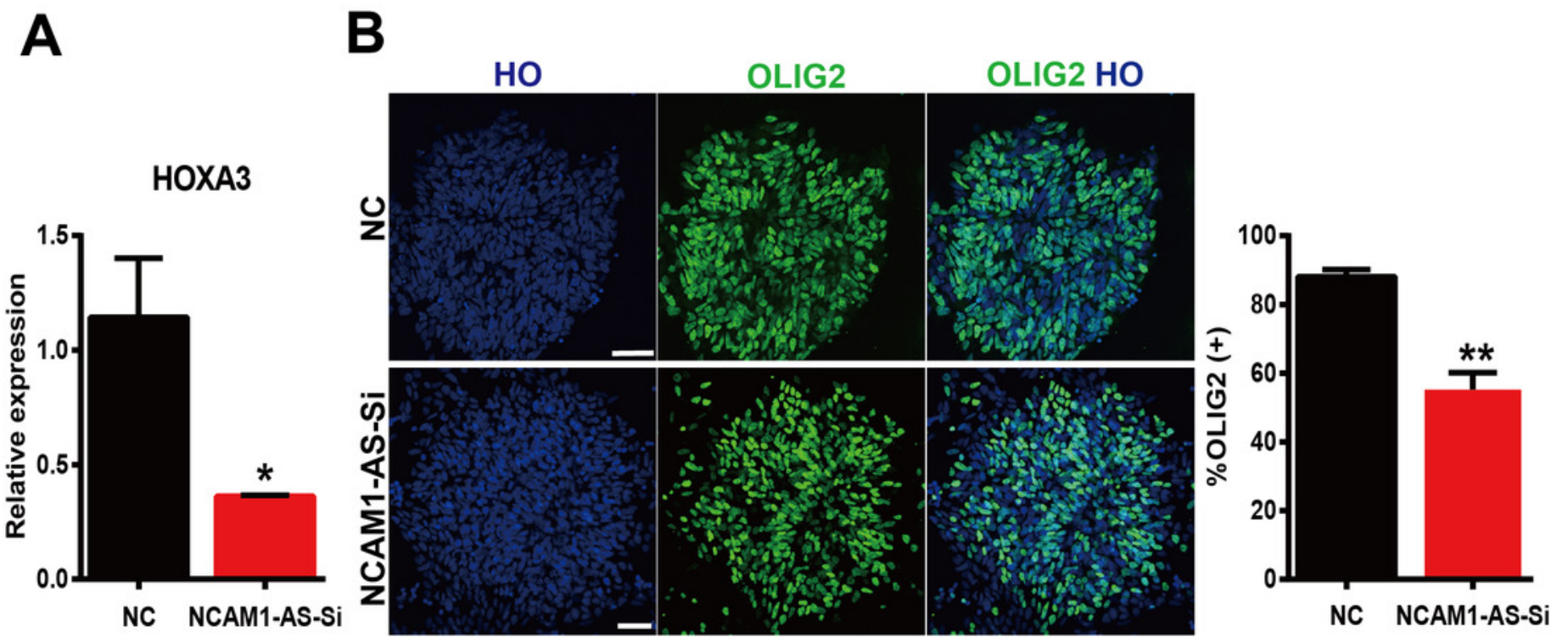

